\title{
New IR imaging modalities for cancer detection and for intra-cell chemical mapping with a sub-diffraction mid-IR s-SNOM
}

\author{
H. Amrania, ${ }^{a}$ L. Drummond, ${ }^{a}$ R. C. Coombes, ${ }^{b}$ S. Shousha, ${ }^{b}$ \\ L. Woodley-Barker, ${ }^{b}$ K. Weir, ${ }^{a}$ W. Hart, ${ }^{a}$ I. Carter ${ }^{a}$ and C. C. Phillips ${ }^{\star a}$
}

Received 2nd November 2015, Accepted 30th November 2015

DOI: $10.1039 / c 5 f d 00150 a$

We present two new modalities for generating chemical maps. Both are mid-IR based and aimed at the biomedical community, but they differ substantially in their technological readiness. The first, so-called "Digistain", is a technologically mature "locked down" way of acquiring diffraction-limited chemical images of human cancer biopsy tissue. Although it is less flexible than conventional methods of acquiring IR images, this is an intentional, and key, design feature. It allows it to be used, on a routine basis, by clinical personnel themselves. It is in the process of a full clinical evaluation and the philosophy behind the approach is discussed. The second modality is a very new, probe-based "s-SNOM", which we are developing in conjunction with a new family of tunable "Quantum Cascade Laser" $(Q C L)$ diode lasers. Although in its infancy, this instrument can already deliver ultra-detailed chemical images whose spatial resolutions beat the normal diffraction limit by a factor of $\sim 1000$. This is easily enough to generate chemical maps of the insides of single cells for the first time, and a range of new possible scientific applications are explored.

\section{Introduction}

The principles of vibrational spectroscopy, (VS), have been established over several decades, and, for much of this time, reliable off-the-shelf systems have been available that are capable of delivering high quality spectroscopic image (SI) datasets for use by the biomedical community. These datasets have potential utility over a wide range of biomedical disciplines that include histopathology, cytology, surgical targeting and drug discovery. ${ }^{1}$

Very recently, technical advances in array detectors and in tuneable "Quantum Cascade laser" (QCL) narrow line sources have opened up new lines of

${ }^{a}$ Physics Dept., Imperial College, London, SW7 2AZ, UK. E-mail: Chris.phillips@imperial.ac.uk; Tel: +44 (0)207 5947575

${ }^{b}$ Department of Cancer and Surgery, Faculty of Medicine, ICTEM, Room 145, Du Cane Road, London W12 oNN, $U K$ 
experimentation, and most would agree that the mid-IR part $(\sim 3 \mu \mathrm{m}<\lambda<\sim 10 \mu \mathrm{m})$ of the spectrum is experiencing, in technology terms, a renaissance. On the other hand, the reality is that take-up of these technologies by the biomedical scientists themselves, particularly clinicians, is still modest.

This paper starts by examining possible reasons for this, (with the aim of stimulating discussion), to set a context within which IR SI implementations may be evaluated for their genuine biomedical potential. It concludes by using this context to examine two new VS-based imaging technologies that we have developed. They are at roughly opposite ends of the labyrinthine "translational pathway" that, ideally, can take a technology from the physical science research lab into the clinic.

The first example, the so-called "Digistain" imager, is a very simple, strippeddown, SI system that is currently at the clinical validation stage. It arose when, rather than asking the usual scientists/technologists question, i.e. "How can I apply my new research technology in a new and hopefully useful way?" we tried inverting the argument and, instead, asked the question "Can we use our research expertise to develop a system that really could end up being used by clinicians?" Over the course of a 10 year multidisciplinary development programme, we found that this second question demands a far more considered and complex answer than the first. Hopefully we will show how well such a simple incarnation of SI technology can perform in a genuine sharp-edged clinical trial environment.

The second example is a new, probe-based, SI system that is very expensive and, at the moment, very difficult to use. It is formed by marrying an existing high-end commercial s-SNOM (scattering-type Scanning Near-field Optical Microscope) machine to an array of newly available QCL diode lasers. It can deliver SI datasets down to a $\sim \lambda / 1000$ spatial resolution of $\sim 10 \mathrm{~nm}$, i.e. sufficient to generate chemical maps inside a single cell for the first time. It came into being in a more traditional way, i.e. by asking the first of the two questions discussed above. Although we believe it has the potential to deliver game-changing imaging performance across the biosciences, it is at a very early stage, and at the moment we can only give a selection of demonstration images that hint at its potential in the biomedical arena.

\subsection{Issues affecting the translation of VS technologies into the biomedical arena}

There are several issues that came into prominence while we were trying to answer the "Can we use our research expertise, to develop a system that really could end up being used by clinicians?" question, and we choose to list them here. Some may seem obvious, but we generally underestimated their importance, and often we chose to ignore them until it became clear that to do so was no longer tenable. In retrospect, we believe that, if one is genuinely interested in seeing VS technology applied in a biomedical setting, much effort and resource can be saved if one is prepared instead to face these issues as early and as honestly as possible.

1.1.1 Operator dependence and reproducibility. The central question here can be framed as "Can you genuinely envisage an incarnation of your technology that could be used by a clinician, and in a way that would give the same answer every time?" A more dramatic way to put it might be "Would you stake your life on the reproducibility of your technology?" This outwardly simple technical question is often the show-stopper because regulatory procedures will demand that you can 
prove that you can answer it in the affirmative. It is a purely technical question, and long before regulatory approval becomes an issue, you will have to convince investors that, at some stage, you will be able to deliver a positive answer to it. If they cannot be convinced, all commercialization prospects will wither, and no matter how powerful the technology is, it will simply never leave the developers lab.

Because this issue is so central, simplified and well-understood data acquisition and processing protocols become paramount. This is true even if, to technologists, they appear to be technically inferior to more sophisticated approaches. Only the former can be heuristically tested, and then tightly specified, and written into a so-called "Standard Operating Procedure".

This "SOP" document is critical and it has to be able to "lock-down" the procedures in a way that limits variability to acceptable levels when the measurements are performed with different machines, in different labs, and by different people. A successful trial should not be thought of as validating a technology, but rather it validates a technology paired with a SOP. For VS-based technologies, one of the biggest problems comes with so-called "unsupervised" SI data processing methods, because variations, e.g. in image uniformity or signalto-noise (SNR) ratio, can have completely unpredictable effects on the data components that the software settles on in the course of its data analysis. ${ }^{18}$

1.1.2 "Occam's razor" and the quest for technology validation. Vibrational spectroscopies and SI technologies deliver huge and rich datasets, and this is often cited as one of their strengths by the scientific community. In most practical applications however, the overwhelming majority of this data will have to be discarded and the central question then becomes how this should be achieved.

In clinical settings the degree of data reduction becomes especially extreme. Medical practitioners are already factoring in huge quantities of "soft" information when they, say, reach a diagnosis, or decide on a patient's treatment plan. As a consequence, any new technologies that hope to augment the clinical process will likely have to boil an extremely complex informational dataset down to a simple yes/no decision, or, at most, a number on a scale of 1 to 10 . If this is not done, the data is unlikely to be of realistic use to a clinician.

The problem with SI technologies is the huge, (effectively infinite) parameter space. There are so many potential ways to reduce the SI data that a form of data paralysis can take hold. A chicken-and-egg problem can set in when it becomes clear that it will be impossible to explore all the possible ways of reducing the dataset to find the best one.

For clinical applications this problem is compounded by the fact that the details of the SOP have to be completely "locked down", (and given adequate IP protection), before any clinical trials can be started. Even slight changes to the SOP will be deemed by the clinical profession to invalidate previously acquired trial data that were taken with the previous SOP. The result is that one has to "nail ones colours to the mast" as early as possible.

All these factors, coupled with the fact that resources are always limited in realistic development programmes, mean that the way forward is likely to involve a substantial degree of educated guesswork and/or some very pragmatic decision making. Ideally this will be guided by a knowledge of the physical/chemical/ physiological mechanism that is underpinning the discriminating or diagnostic power of the SI dataset. Often though this understanding is either partial or completely absent. In this case, decisions can be just as usefully driven by 
technological considerations, such as the limitations of available IR sources and detectors.

1.1.3 Giving biomedical professionals VS-based information in a form they can use. The point has already been made that clinicians are already factoring large amounts of information into their clinical decisions. Most of this is qualitative, and is generated by the clinicians themselves, via their own senses. Very little of it is couched in the language of numbers favoured by scientists and engineers, and most clinicians reach their decisions almost entirely from data that reaches them in the form of images. This is unlikely to change in the foreseeable future.

Moreover, in most cases (e.g., conventional X-ray images, biopsy microscopy, CAT scans, ultrasonic images, MRI etc.), the image data is evaluated almost entirely by eye. The clinician does not, (and does not need to) understand e.g. the contrast mechanisms, or spectroscopic details that generated the image, in order to be able to exploit its clinical value. Even if the images are available in a reliablycalibrated digitized format (and usually they are not), it is unusual for any numerical data to be extracted from them for diagnostic purposes; perhaps the measurement of a physical dimension, or some single-digit index figure.

Taken together, these facts argue strongly for the VS community to concentrate on ways of presenting their spectroscopic information to clinicians in a simple, clear, visual way, probably sweeping all the technical details into the background.

1.1.4 Technology innovation vs. "unmet need". At some point, perhaps halfway along the metaphorical "translational path", the language of the debate changes abruptly from issues that can be characterized by questions like "How good is this technology, say compared with the competition? What new things can it do?" that initially drives we technology developers, to a "What existing problem can it actually solve?" issue. Thenceforward, funders, investors and users all focus on the so-called "unmet need", i.e. the problem, and one that is agreed by the users as being an existing and important one, which the new technology can reasonably hope to address.

The key point is that the "unmet need" exists entirely independently of the technology. It was probably there even before the technology was developed. It is defined by consensus of the potential users of a technology, and not by our community of technology developers.

If such an "unmet need" can be identified early on it can have a dramatic effect on the trajectory of a technology and save much wasted effort. This may seem so obvious as not to need saying, but the reality is that many, if not most, Vs-based technology development programmes, target an "application" that was chosen, sometimes even invented, by the technology developer themselves. Usually this is done without sufficient (or, sometimes, any) discussion with potential end users.

\section{The "Digistain" IR imager, for cancer detection and diagnosis}

\subsection{Background and the "unmet need"}

At the moment, $99 \%$ of cancers are detected and characterised by excising a small "biopsy" tissue sample from the patient, drying it, slicing it thinly $(\sim \mu \mathrm{m})$, and staining it with two vegetable dyes that the histopathology community settled 
upon over 100 years ago. Haematoxylin stains the acidic parts of the specimen (i.e. the cell nuclei) blue, and eosin stains the cytoplasmic proteins pink. A trained histopathologist then "grades" this "H + E" specimen by eye, in a standard optical microscope. These "grades" often inform life-critical treatment decisions. Although the grading SOP protocols vary between different cancers, always the judgements are subjective, and often there is poor concordance between histopathology practitioners. Considered worldwide, this constitutes a very significant "unmet need".

\subsection{The physical principles behind "Digistain"}

Our Digistain imager uses the physics of mid-IR absorption in the same excised and dried tissue slice as a quantitative way of mapping out the ratio of phosphate moieties, found predominantly in cell-DNA, ${ }^{2}$ to the proteins in the cell cytoplasm. The chemical concentrations are measured by taking a set of calibrated images in four mid-IR bands ${ }^{2}$ (Fig. 1). The resulting chemical map is converted into a computer generated false colour image $\mathrm{e}^{3}$ and presented to the histopathologist in a way that allows it to be used to augment and inform the process of tissue grading.

The idea seems simple, but in execution it presents significant technical challenges. Reproducibility requires that the effects of non-uniformity and nonlinearity in the detector array, together with variations arising from non-uniform sample illumination, and fluctuating thermal background signals, all have to be identified and engineered out. ${ }^{4}$ Also the 4 images, which are taken at widely separated wavelengths, have to be accurately calibrated and registered for the ratioing process to work. Finally the optical throughput and image acquisition has to be refined to the point where adequate SNR images can be obtained with practical acquisition times of only a few minutes.

\subsection{Factors affecting likelihood of successful clinical application}

The choice of these two particular chemical moieties for Digistain was determined by a mix of the factors outlined in the previous section. In the first place, they give prominent features in the IR spectra that are within reach of a combination of comparatively basic (i.e. uncooled) mid-IR emitters and detectors. This added to their attractiveness as the features of the dataset most worthy of preserving in the face of the "Occam's razor" principle (Section 1.2.2).

Secondly, these features provided an image that could be thought of as being an approximate analogue of the existing $\mathrm{H}+\mathrm{E}$ one. The concentration of the phosphate moiety correlates with the acidic components in the tissue that turn out blue in the $\mathrm{H}+\mathrm{E}$ images, and the amide moiety correlates with eosinophilic components of the tissue that stain pink. This way we aimed to reduce the SI image data to a form that was "pre-digested" into a format that was best suited for the histopathologists to assimilate and use, in line with the considerations of Section 1.2.3.

Thirdly, we only settled on this subset of the possible SI data after having already undergone a science-driven research programme consisting of several years of technologically complex laser based spectroscopy. ${ }^{5,6}$ Although this early study itself did little to establish a route to a viable clinical application, it had involved numerous consultations with histopathology professionals. These early 


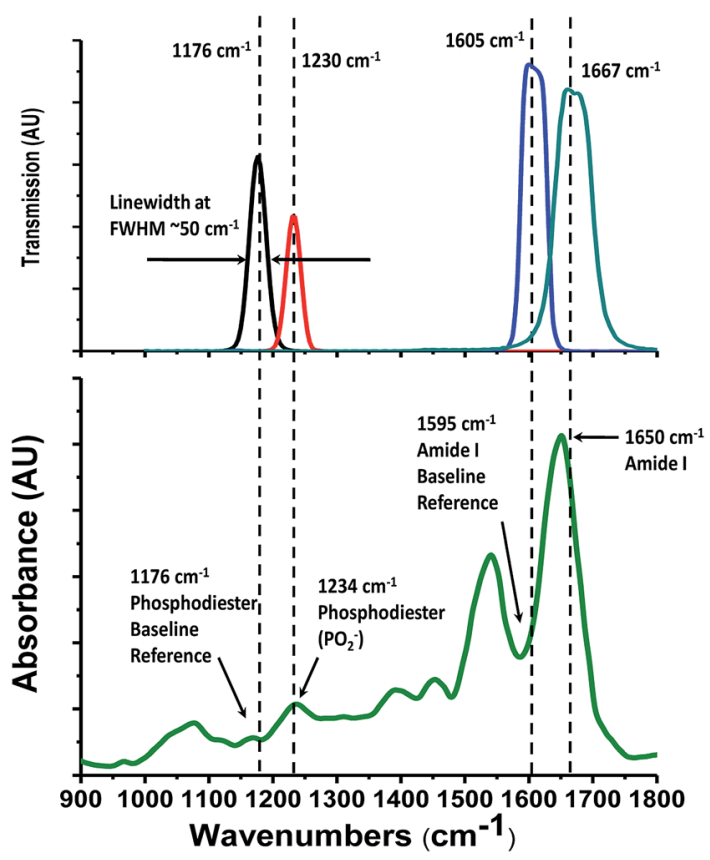

Fig. 1 Lower trace, a typical absorption spectrum of dried, unstained, human biopsy tissue, in this case, a $2.5 \mathrm{~mm} \times 2.5 \mathrm{~mm}$ section of an oesophageal biopsy section, initially harvested using a standard "FFPE" (formalin fixed paraffin embedded), protocol, then subsequently deparaffinised. It is recorded at $4 \mathrm{~cm}^{-1}$ spectral resolution with an FTIR microscope. Upper trace, transmission spectra of the 4 bespoke mid-IR bandpass filters used in the "Digistain" imager. They work in pairs to acquire calibrated signal and baseline images at wavelengths corresponding to absorption by the amide I and phosphodiester chemical moieties.

consultations allowed us to identify and roughly target an important histopathology "unmet need" (Section 1.2.4), and steered the design principles behind the prototype imager of Fig. 2.

Fourthly, although there are of course many types of cancers it is well known that, in the majority of them, the cell reproduction cycle becomes significantly disrupted as healthy tissue becomes diseased. This is reflected in the fact that histopathologists frequently employ measures of both "pleomorphism" (changes in the relative size and shape of the cell nuclei) and "mitotic activity" (the rate of cell division) as key factors in their tissue grading SOPs. The "Digistain Index" (DI) that our imager measures is proportional to the ratio of concentrations of the phosphate to amide moieties, so we had grounds for hoping that a robust way could be found to correlate this data with tumour grade. ${ }^{7-9}$ In other words we were using an educated guess (Section 1.2.3) to prejudge the data reduction process that would generate the most useful images, before committing to large-scale clinical trials.

Fifthly, with Digistain we were focussed on an optical measurement, i.e. midIR absorption in a smooth-sided tissue slice, which we could reasonably hope to be able to make with good robustness and reproducibility (Section 1.2.1). By taking an appropriately scaled and background corrected ratio ${ }^{3}$ of the absorption 


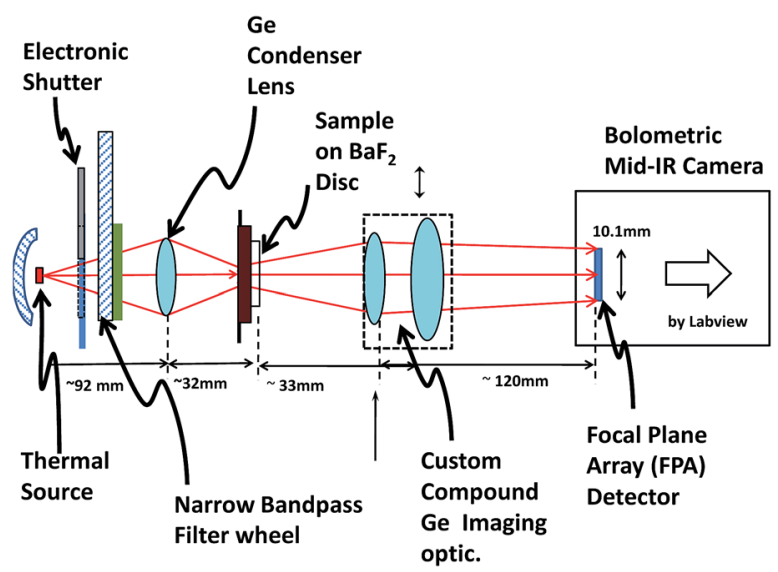

Fig. 2 Schematic of fully automated "Digistain" IR imager for histopathology. At the base (LHS) of the unit is a broadband thermal IR source with an electronically controllable shutter. The IR beam then passes through a set of bespoke, computer-selectable, narrowband IR filters, and then through a germanium condenser lens carefully designed to maximize the optical throughput. The biopsy sample is mounted on a $25 \mathrm{~mm}$ diameter $\mathrm{BaF}_{2}$ disc and is imaged, through a custom designed low aberration imaging optic, onto a bolometric focal plane array with a magnification of $\times 3.1$ and at a diffraction-limited spatial resolution. The instrument has a $30 \times 30 \mathrm{~cm}$ bench footprint, requires only a single $13 \mathrm{~A}$ socket, and can be operated, with a single mouse click, by untrained personnel.

of the moieties (Fig. 3) we could design a new SOP (3) that would be least sensitive to operator-dependent factors. The latter are mostly due to variations in the thickness of the tissue slice, but there is also the issue of fixing protocols, which can vary from site to site.

Sixthly, the Digistain approach presents the mid-IR spectroscopic information visually, and in a way that can readily be compared with existing $\mathrm{H}+\mathrm{E}$ slides

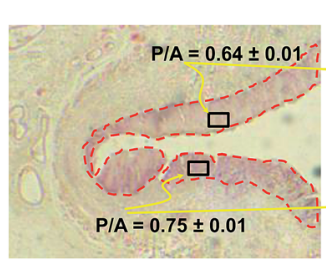

(a)

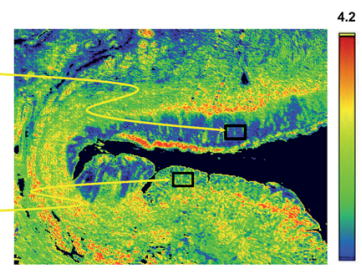

(b)

Fig. 3 Example of the discriminating power of a Digistain image, in this case of an oesophageal biopsy. Left: Conventional " $\mathrm{H}+\mathrm{E}$ " stained biopsy section. The regions delineated with red dotted lines are epithelial tissue, and the upper one is healthy (as judged by a trained histopathologist), whereas the lower right one is dysplastic. Right: Corresponding "Digistain" image, taken from an unstained slice of tissue directly adjacent, on the FFPE tissue block, to the one used for the $\mathrm{H}+\mathrm{E}$ stain. It is a false-colour image constructed from the measured "phosphide-to-amide" ratio, P/A, extracted from the midIR images, as described in Fig. 1 and 2. The healthy tissue shows up blue, and the dysplastic tissue green. Averaging this "P/A ratio" (which we call the "Digistain Index", DI), over the pixels in the two black rectangular areas gives numerical values for the two tissue types that differ at the $>33 \sigma$ level. 


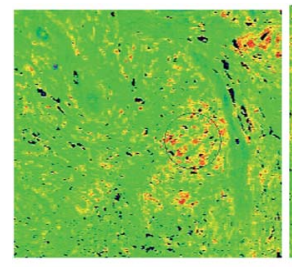

(a)

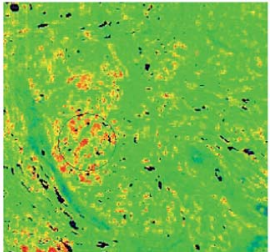

(b)

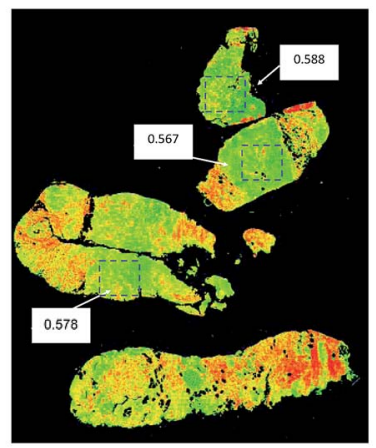

(c)

Fig. 4 Examples of Digistain reproducibility test. (a) and (b) are images of the same slice of breast biopsy tissue, but with the sample rotated and translated between scans. The circled areas are the same portion of tissue in each case, and return "Digistain Index" values of $1.0667 \pm 0.009$ and $1.061 \pm 0.009$ ( $3 \sigma$ limits), implying a technical reproducibility better than one part in 100. (c) contains three different core biopsies taken simultaneously from the same histological "grade 2" breast tumour. The boxed green areas were identified as tumorous by a trained histopathologist on the basis of the corresponding $\mathrm{H}+\mathrm{E}$ slides. The "Digistain Index" here is reproducible to $\mathrm{DI}=0.576 \pm 0.089$ across different parts of the tumour.

(Section 1.2.3). There is an important point here; because the $\mathrm{H}+\mathrm{E}$ slides are the clinician's so-called "gold standard" they will continue to be used in any event. No clinician would risk running against the grain of the profession by attempting to diagnose a condition as serious as cancer using only a revolutionary and untested technology. Often we are asked if Digistain can be adapted for in vivo use and the answer, in the short term at least, is no, at least not without a major engineering programme on broad spectrum mid-IR compliant imaging endoscopes. However, in some ways this can be seen as an advantage rather than a shortcoming. If we had, say, produced a machine that required an extra in vivo endoscopy procedure on top of the established standard biopsy one, it would probably have been much harder to test it (it would have needed separate live patient trials) in order to get it adopted.

A critical factor in Digistain's favour is that after the $\mathrm{H}+\mathrm{E}$ biopsies have been examined, the excised tissue samples are chemically preserved in wax (which can easily be dissolved away many years later), and archived, along with the original $\mathrm{H}+\mathrm{E}$ slide, and a full medical history. There are millions of documented specimens of all types of cancer that one can go back to and study 


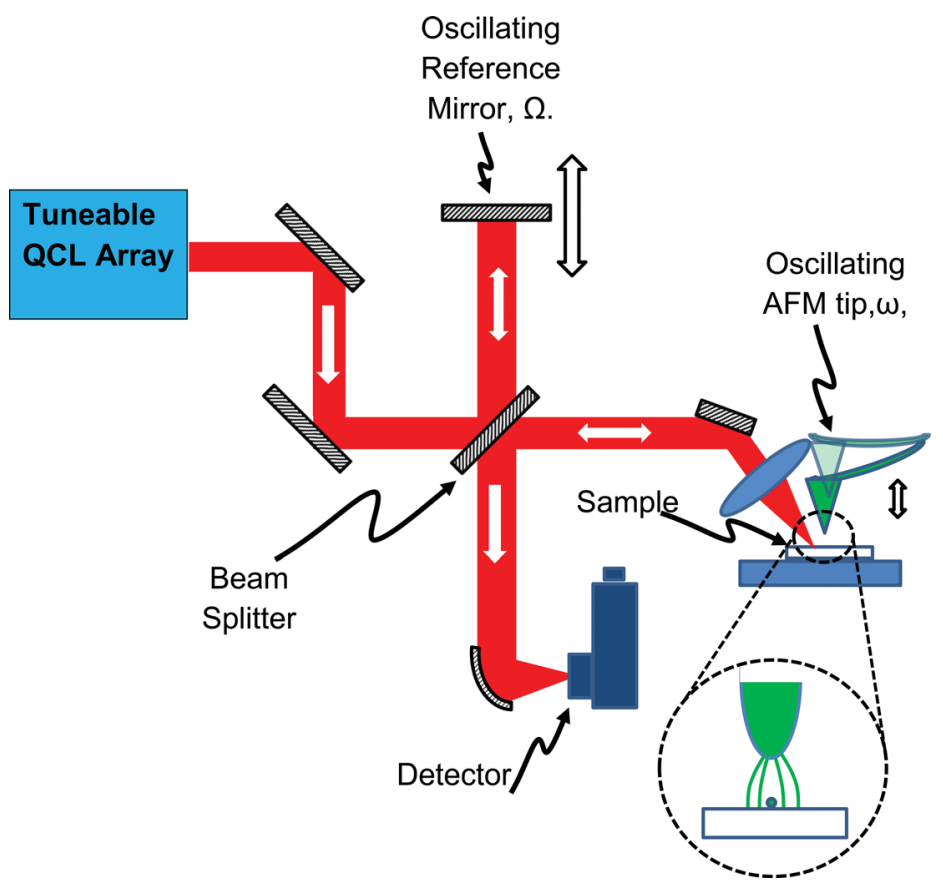

Fig. 5 Schematic of the mode of operation of a "scattering-type Scanning Near-field Optical Microscope" (s-SNOM). An AFM tip hovers close to the sample surface and vibrates, vertically, at a frequency $\omega$. IR laser light is focused onto the tip region, and concentrated, into a spatial area roughly corresponding to the tip diameter (inset), by the "lightning rod" effect. By detecting the portion of the backscattered light fluctuating at $3 \omega$ (i.e. a harmonic of the tip vibration), the light backscattered from this small region can be isolated. An interferometer (with a reference mirror oscillating at $\Omega \ll \omega$ ) is used to measure both the amplitude and phase of the backscattered light, from which the optical properties of the very small sample area that is interacting with the AFM tip can be inferred. An image is assembled by rastering the AFM tip across the sample in the usual way.

with Digistain. This means it can be tested and validated with logistical and ethical hurdles that are far lower than if one had to secure access to cohorts of new live patients to trial it with. Of course the issues of reliability and reproducibility are just as key here; they require longitudinal trials to establish the degree of chemical stability, specifically, as judged by our new imaging method, afforded by the FFPE method (Fig. 4).

At present we are nearing the end of a clinical trial, involving upwards of 200 breast cancer patients. They were assessed according to the RECIST criteria (a set regulated assessment criteria that all breast cancer diagnostic methods must follow), and the biopsies were fixed using the RCP (Royal College of Pathologists) fixing protocol. To carry weight with the medical profession, these trials have to be performed, by a large team, under strict conditions of anonymity, double-blindness and with suitably experienced and accredited statistical analysis staff. Unfortunately, for this reason, we are not allowed to discuss the results at all until they have been formally released, but we hope to be able to discuss this in more detail at the meeting. 

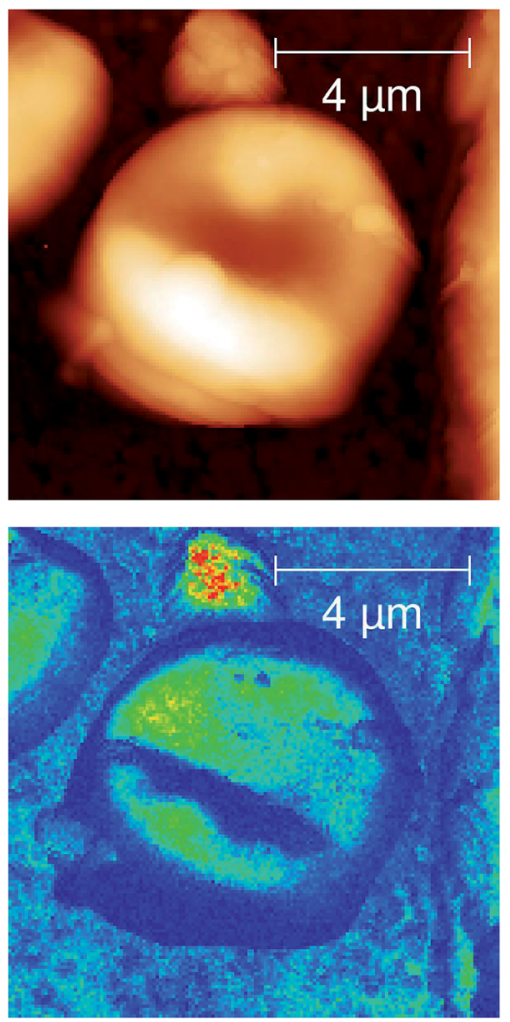

Fig. 6 Upper; AFM scan of the physical structure of a single red blood cell in a squamous epithelial oesophageal biopsy. Lower image, s-SNOM 3rd harmonic image, taken at $\lambda=$ $9.81 \mu \mathrm{m}, \sim 20 \mathrm{~min}$ scan time and $67 \mathrm{~nm}$ pixel resolution. The resolution here is $\sim 140$ times better than the diffraction limit at this wavelength and has allowed us to image chemical contrast within the cell for the first time.

\section{A tuneable s-SNOM for intracellular chemical mapping}

\subsection{Introductory comments}

Typical cells are 10s of $\mu \mathrm{m}$ across, and although $\sim \mu \mathrm{m}$-level diffraction-limited resolution mid-IR imaging is possible (5) SNR limitations mean that it is only practical with sophisticated bench-scale laser sources. These are very challenging to implement, and they still fail to deliver enough resolution to "look inside" a single cell. In principle, Raman methods can deliver sub-micron resolution, but the inefficiency of the Raman signal generation process means that getting useful signals from beams that are focussed so tightly involves power densities that are likely to compromise biological material.

The advent of a cell-level chemical analysis capability would have a wide ranging impact across the biosciences. At present, chemical analysis of changes to cell chemistry involve generating a sample of a large number of cells, (say by flow cytometry or cell breeding), breaking them up, and then analysing the mix with any one of a number of chemist's tools, including wet-chemistry assay, IR 


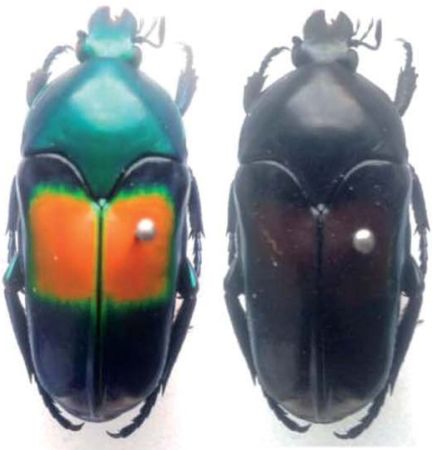

Faraday Discussions

(a)

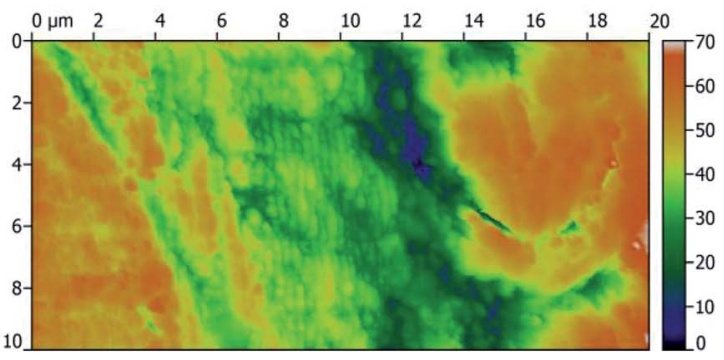

(b)

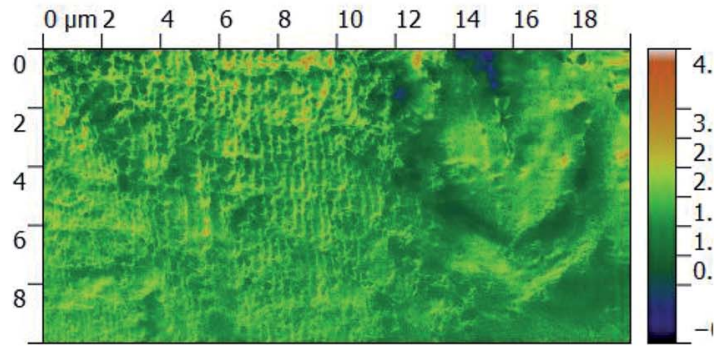

(c)

Fig. 7 (b) AFM topography and (c) $\lambda=10.21 \mu \mathrm{m} \mathrm{s}$-SNOM scattered amplitude image of a "natural photonic crystal", i.e. the shell of an iridescent beetle (a) that has in-built periodic structure that gives it a chiral light scattering characteristic which means its shell markings only show up under circularly polarised light. The s-SNOM image has given the chemical contrast of the components that make up the multilayers in the insect shell. In order to generate the shell's interference features in the visible part of the spectrum, the concentrations of these components vary on a sub-micron length scale, and only the sSNOM can deliver chemical images at this resolution.

spectroscopy, mass spectroscopy or labelling methods. Of course, all these "looking at the bits" approaches destroy the cell morphology information, and they necessarily average over any cell-to-cell variation in either chemistry or morphology.

Real cell-level imaging would have a major impact across the biosciences. The cell-to-cell variations within a population could give crucial insights into $e$.g. how cancer starts, (often with a single rogue cell), and, generally speaking cell-level pathology could be radically transformed. In pharmacology, the question of "How 


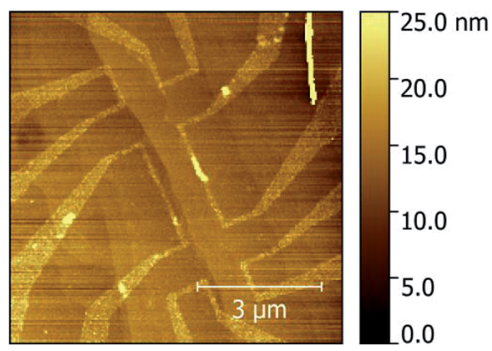

(a)

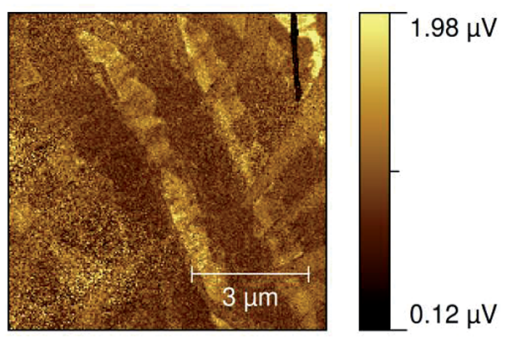

(b)

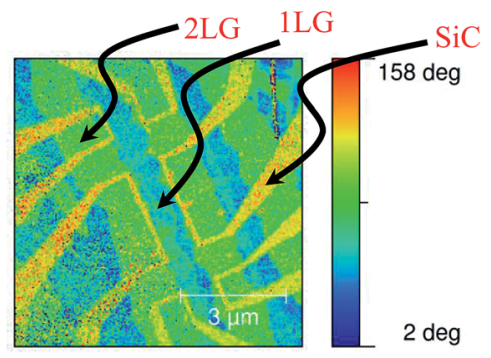

(c)

Fig. 8 (a) AFM topology, and $\lambda=10.3 \mu \mathrm{m} \mathrm{s}$-SNOM amplitude (b) and phase (c) images of an FET transistor fabricated from a monolayer graphene (1LG) with occasional bilayer inclusions (2LG) sheet, etched away in places to reveal the SiC substrate.

does this drug act?" often boils down to "Where, and for how long, does this drug bind?" and being able to answer the latter, at a cell level, could have major implications for the drug discovery industry.

In this context, ultra-high-resolution imaging offers a unique "concentration advantage" feature. Fortunately, many pharmacologically active compounds have distinctive vibrational spectra, but most are active at mean concentrations in the 1 part in $10^{9}$ region. Obviously this presents a major sensitivity challenge for VSbased technologies. If the drug binds at a particular site though, the local concentration can be dramatically elevated, and if the SI resolution is increased to the point where only a comparatively small number of molecules can be resolved, this effect can, in principle, lift the signal out of the noise.

So-called s-SNOMs promise to deliver this information. ${ }^{\mathbf{1 0}}$ They work by focussing mid-IR radiation onto the point where the probe tip of an atomic force microscope (AFM) touches a sample, and then analysing the degree to which the radiation is backscattered. The sharpness of the AFM tip concentrates the optical 
fields, via a "lightning rod" effect, into an area roughly commensurate with the AFM tip diameter. They offer spatial resolutions down to $\sim 10 \mathrm{~nm}$ even with $\lambda \sim 10$ $\mu \mathrm{m}$ light, and they work with radiation densities and photon energies that are both low enough to be completely compatible with biological material.

However, the backscattered signal is small, and it requires a sophisticated interferometric signal recovery scheme ${ }^{\mathbf{1 1}}$ (Fig. 5) to pick it up. In turn this necessitates a coherent IR source. Usually this is a $\mathrm{CO}_{2}$ laser that can only be tuned over a spectral range that is too narrow for useful IR spectroscopy. Until recently the only broad-band alternative source was a complex lab-scale laser system that used non-linear frequency mixing technology, ${ }^{12}$ or an IR synchrotron, ${ }^{13}$ but now the situation is rapidly evolving with the advent of commercially available "Quantum Cascade Lasers" (QCLs).

3.1.1 Brief synopsis of "Quantum Cascade Laser" technology. QCLS are a new type of laser diode ${ }^{14}$ that use sophisticated semiconductor nano-structuring to produce a device that can be designed to lase across a wide wavelength range, from the mid-IR to the THz part of the spectrum. The technology is rapidly evolving, but at the moment, practical (i.e. operating at close to $300 \mathrm{~K}$ ) commercially available devices roughly span the $5 \mu \mathrm{m} \sim \lambda \sim 11 \mu \mathrm{m}$ range.

However, each device typically tunes through 2 or $3 \mu \mathrm{m}$, so covering the normal mid-IR VS range is expensive because it needs 4 or 5 devices to be engineered into a single system. CW QCL operation is only available in parts of the spectrum, but they generate $\mathrm{CW}$-equivalent powers in the $\mathrm{mW}$ range, with very narrow linewidths and close to diffraction-limited beam quality. The commercial QCL systems now offer turn-key operation, coupled to a convenient computercontrolled tuning system that makes them very easy to use.

Against this however, many of the QCL designs use external cavities for tuning the lasers, and these are extremely sensitive to small amounts of light being scattered back from the s-SNOM interferometer. This makes it difficult to get them to work stably enough to use with the s-SNOM. Also, the fact that some wavelengths are only available as pulse trains introduces technical complications to the signal chain ${ }^{\mathbf{1 5}}$ (as well as for imaging applications more generally) and in practice, when the QCL system switches between different laser devices as it is tuned, the s-SNOM optics usually have to be manually re-aligned.

3.1.2 Quantum Cascade Lasers applied to s-SNOM. Our s-SNOM system (NeaSNOM, Neaspec) uses probes with $\sim 10 \mathrm{~nm}$ tip radius, oscillating at frequencies in the 50-300 kHz region. A range of QCLs are used, (Lasertune, Block Engineering, and MirCat, Daylight Solutions), both in CW and pulsed modes. When the lasers can only operate pulsed, steps have to be taken to synchronise the laser pulse rate to the sampling rate of the circuitry that digitises the detector signal, in order to avoid introducing large amounts of digitisation noise. ${ }^{15}$

Depending on the application, the backscattered light is polarised before being detected. In "plasmonic" experiments s-polarised incident light is used, because it can excite plasmonic modes in metal nanostructures without being sensitive to chemically based contrast mechanisms that arise between the different material components (usually a metal and a dielectric) that are used to make the nanostructure. If the detection channel is then p-polarised, (the socalled "cross polarisation scheme") it selects only the p-polarised field components that arise from the excitation of plasmons in the nano-photonic device, and is largely immune to chemical contrast effects. 
One way to understand this is to notice that the incoming s-polarised light excites the resonant in-plane plasmon modes in the sample, without itself significantly interacting with the probe (which is a very poor antenna to anything but light polarised perpendicularly to the surface). For most biomedical experiments, it is the chemical contrast that is of interest, so both incoming and backscattered channels are p-polarised, as this is the polarisation that interacts with the tip and the sample most strongly (Fig. 5 inset). In all cases great care needs to be taken to avoid light backscattered from any of the optical components in the setup from reaching the QCL.

3.1.3 Demonstration images from the QCL s-SNOM. As well as the optical image, the s-SNOM can be operated as a standard AFM, and Fig. 6 shows the morphological information of a blood cell, taken from a deparaffinised FFPE biopsy slice, similar to those used in the Digistain experiments of Section 2. The lower trace is an early s-SNOM image, taken at a wavelength where stable QCL operation was possible. It's recorded at a $67 \mathrm{~nm}$ pixel resolution, and clearly records the cell structure at a $\sim \lambda / 140$ resolution.

From the field of natural biophotonics, there are a number of iridescent beetle species that have evolved with periodic "photonic crystal" chiral shells, which give them distinctive markings that are visible only in circularly polarised light (Fig. 7). The question arises as to whether the chirality behind this is an accidental "self-organisation" effect arising from the natural chirality of the biomolecules that are forming the periodic structure in the shell, or whether the chirality has been generated by evolutionary forces. Fragments of the shell were polished to yield a smooth surfaced sample with minimal morphological contrast in the AFM image. The s-SNOM image however uses the chemical contrast to reveal the periodic structure of the chitin helices thought to be the origin of the effect.

Fig. 8 images a field effect transistor (FET) device fabricated for a graphene sheet grown epitaxially on a SiC substrate. ${ }^{16,17}$ The AFM morphology image reveals the device structure. The s-SNOM phase image, in this example, approximately corresponds to the absorption in the graphene layer, and reveals regions of differing thickness, and the edge effects responsible for the device operation.

At the time of writing the QCL s-SNOM system is being reengineered to facilitate tuneable, full-spectrum spectroscopic imaging. The aim is to substantially increase the amount of scientific information that can be inferred from these images, and we hope to have new data to present at the meeting itself.

\section{Conclusions}

We have presented here two new ways of using mid-IR chemical imaging, and in both cases, as befits the topic of this discussion meeting, the focus has been on what they can offer the biomedical community. They are both at very different stages of technical readiness, and they face very different challenges in the future.

For Digistain the technical hurdles have been overcome, the clinical potential has essentially already been proven; the challenge now is to validate it using criteria and methods that will engage the support and interest of the clinicians. This is already underway, but we are well aware that it will have to be achieved on a wide enough scale to convince investors to produce them as tools to put in the medics' hands, because without that, the idea will come to nothing. 
For the s-SNOM it is the beginning, and the challenges are still mostly technical. We believe it has a very bright future as a new scientific research tool for a wide range of biomedical problems, but, already, we are on the lookout for that elusive "unmet need".

\section{Acknowledgements}

Financial support from the UK Engineering and Physical Science Research council (grant numbers EP/K503733/1, EP/G031819/1 and EP/K 029398/1) is gratefully acknowledged. The Graphene FET samples were kindly supplied by Olga Kazakova and Vishal Panchal. We thank The Imperial Biomedical Research Centre and The Imperial Experimental Cancer Medicine Centre for technical assistance.

\section{Notes and references}

1 Infrared and Raman Spectroscopic Imaging, ed. R. Salzer and H. W. Siesler, 2009, Wiley-VCH, Weinheim, ISBN 978-3-527-31993-0.

2 H. Amrania, G. Antonacci, C.-H. Chan, L. Drummond, W. R. Otto, Nicholas A. Wright and C. Phillips, Opt. Express, 2012, 20(7), 7290-7299.

3 C. C. Phillips and H. Amrania, Infrared Imaging of Biological Material, G B Patent, filed 29, April 2015, Application number PCT filed GB1507314.1.

4 L. Drummond, PhD thesis, Imperial College London, 2014.

5 H. Amrania, A. McCrow and C. Phillips, Rev. Sci. Instrum., 2009, 80, 123702.

6 H. Amrania, A. P. McCrow, M. R. Matthews, S. G. Kazarian, M. K. Kuimova and C. C. Phillips, Chem. Sci., 2011, 2(1), 107.

7 L. M. Merlo, L. S. Wang, J. W. Pepper, P. S. Rabinovitch and C. C. Maley, Adv. Exp. Med. Biol., 2010, 676, 1-13.

8 C. N. Battlehner, P. H. Saldiva, C. R. Carvalho, T. Y. Takagaki, G. S. Montes, R. N. Younes and V. L. Capelozzi, Histopathology, 1993, 22(1), 31-34.

9 S. J. Jang, J. M. Gardner and J. Y. Ro, Adv. Anat. Pathol., 2011, 18(2), 165172.

10 F. Keilmann and R. Hillenbrand, Philos. Trans. R. Soc. London, Ser. A, 2004, 362, 787.

11 N. Ocelic, A. Huber and R. Hillenbrand, Appl. Phys. Lett., 2006, 89, 101124.

12 F. Huth, A. Govyadinov, S. Amarie, W. Nuansing, F. Keilmann and R. Hillenbrand, Nano Lett., 2012, 12(8), 3973-3978.

13 H. A. Bechtel, E. A. Muller, R. L. Olmon, M. C. Martin and M. B. Raschkeb, Proc. Natl. Acad. Sci. U. S. A., 2014, 111(20), 7191-7196.

14 J. Faist, F. Capasso, D. L. Sivco, C. Sirtori, A. L. Hutchinson and A. Y. Cho, Science, 1994, 264, 553-556.

15 E. Yoxall, M. Navarro-C, M. Rahmani, S. A. Maier and C. C. Phillips, Appl. Phys. Lett., 2013, 103, 213110.

16 V. Panchal, R. Pearce, R. Yakimova, A. Tzalenchuk and O. Kazakova, Sci. Rep., 2013, 3, 2597.

17 V. Panchal, A. Lartsev, A. Manzin, R. Yakimova, A. Tzalenchuk and O. Kazakova, Sci. Rep., 2014, 4, 5881.

18 K. Atkinson, Mid-IR Biological Imaging, MSc thesis, Imperial College London, 2006. 